\title{
EXPONENTIAL DECAY OF WEAK SOLUTIONS FOR HYPERBOLIC SYSTEMS OF FIRST ORDER WITH DISCONTINUOUS COEFFICIENTS $\left({ }^{1}\right)$
}

BY

\author{
HANG-CHIN LAI
}

\begin{abstract}
The weak solution of the Cauchy problem for symmetric hyperbolic systems with discontinuous coefficients in several space variables satisfying the fact that the coefficients and their first derivatives are bounded in the distribution sense is identically equal to zero if it is exponential decay.
\end{abstract}

1. Introduction. For hyperbolic systems of first order with sufficiently smooth coefficients, the existence theorem and the uniqueness theorem of solutions for the Cauchy problem were proved by Petrovskir, Leary and others (cf. Gel'fand [3]). Gel'fand [3] proposed the question of what conditions assure the existence and the uniqueness of solutions of the Cauchy problem in the case of discontinuous coefficients. Recently, Conway [1], and Hurd and Sattinger [4] investigated such problems. Conway proved the existence theorem for the case of a single linear equation in several space variables and the uniqueness theorem for a quasi-linear equation. Hurd and Sattinger proved the existence theorem for the case of first order hyperbolic systems in several space variables and the uniqueness theorem in the case of one space variable. They also proved the existence theorem and the uniqueness theorem for hyperbolic equations of second order. On the other hand, Masuda [5] proved a theorem on the exponential decay of solutions for hyperbolic equations with smooth variable coefficients. In this paper we shall prove a result on the exponential decay of weak solutions for first order hyperbolic systems with discontinuous coefficients.

2. Preliminaries and the main result. Let $E^{n+1}$ be the $(n+1)$-dimensional Euclidean space with points denoted by $(x, t)$, where $x$ is a point in the $n$-dimensional Euclidean space and is denoted by its coordinates $\left(x_{1}, x_{2}, \cdots, x_{n}\right)$. Let $\mathfrak{D}$ be the half space $\left\{(x, t) \in E^{n+1} \mid t \geq 0\right\}$. The hyperplane $t=\tau$ is denoted by $H_{\tau}$. We consider two (vector-valued) functions $u=\left(u_{1}, u_{2}, \ldots, u_{l}\right)$ and $v=$ $\left(v_{1}, v_{2}, \ldots, v_{l}\right)$ defined in $\mathfrak{D}$ and put

Received by the editors December 30, 1970.

AMS 1970 subject classifications. Primary 35L45, 35L99.

Key words and phrases. Hyperplane, derivative in distribution sense, weak solution, mollifier method, smoothed functions.

(1) This research was partially supported by Math. Res. Center, Taiwan, Republic of China. 


$$
\langle u, v\rangle=\sum_{i=1}^{l} u_{i} v_{i} \text { and }|u|^{2}=\langle u, u\rangle \text {. }
$$

The space $L^{2}(\mathfrak{D})$ is the Hilbert space consisting of all measurable functions $u=\left(u_{1}, u_{2}, \cdots, u_{l}\right)$ for which

$$
\iint_{\mathfrak{D}}|u|^{2} d x d t<\infty
$$

For brevity we use the notation

$$
(u, u)=\|u\|^{2}=\iint_{\mathscr{D}}|u|^{2} d x d t .
$$

In the following, $f \in L_{10 \mathrm{c}}^{2}(\Omega)$ for a function or a vector-valued function $f$ defined on a region $\Omega$ in some Euclidean space means that $f$ or every component of $f$ is square integrable on every compact subset of $\Omega$.

Now we consider the following Cauchy problem of the first order symmetric hyperbolic system:

$$
\begin{aligned}
& \frac{\partial}{\partial t} u+\sum_{i=1}^{n} \frac{\partial}{\partial x_{i}}\left(A^{i} u\right)+B u+C=0 \\
& u(x, 0)=\psi(x)
\end{aligned}
$$

where $A^{i}(1 \leq i \leq n)$ are $l \times l$ symmetric matrices, $B$ is an $l \times l$ matrix, $C$ is an $l \times 1$ matrix and each element of these matrices belongs to $L_{1 \text { oc }}^{2}(\mathscr{D})$. Moreover, we suppose that $A^{i}, B$ and their first derivatives in the distribution sense are bounded in $\mathfrak{D}$.

A (vector-valued) function $u$ is called a weak solution of the Cauchy problem (1), (2) for the Cauchy data $\psi(x) \in L_{1 \text { oc }}^{2}\left(H_{0}\right)$ if $u \in L_{1 \text { oc }}^{2}(D)$ satisfies (2) and

$$
\begin{aligned}
\int_{0}^{t} \int_{H_{s}}\left[\left\langle u, \frac{\partial \phi}{\partial s}\right\rangle+\sum_{i=1}^{n}\left\langle A^{i} u,\right.\right. & \left.\left.\frac{\partial \phi}{\partial x_{i}}\right\rangle-\langle B u, \phi\rangle-\langle C, \phi\rangle\right] d x d s \\
& +\int_{H_{0}}\langle\psi, \phi\rangle d x-\int_{H_{t}}\langle u, \phi\rangle d x=0
\end{aligned}
$$

for any $t(>0)$ and for any function $\phi \in C^{1}\left[0, \infty ; H_{0}^{1,2}\left(E^{n}\right)\right]$. Here the space $H_{0}^{1,2}\left(E^{n}\right)$ is the closure of $C_{0}^{\infty}\left(E^{n}\right)$ by the norm

$$
\|\phi\|_{1}=\left(\int_{E^{n}} \sum_{|a| \leq 1}\left|D_{x}^{a} \phi\right|^{2} d x\right)^{1 / 2},
$$

and the space $C^{1}\left[0, \infty ; H_{0}^{1,2}\left(E^{n}\right)\right]$ consists of all functions $\phi$ with the following properties: (i) $\phi$ is measurable in $E^{n}$, (ii) for almost all $t \in[0, \infty)$, the func- 
tion $\phi(x, t)$ in $x$ belongs to $H_{0}^{1,2}\left(E^{n}\right)$, and (iii) the norm $\|\phi\|_{1}$ as a function of $t$ belongs to $C^{1}([0, \infty))$.

The theorem which we shall prove is as follows:

Theorem. Suppose that $u \in L_{1 \text { oc }}^{2}(D)$ is a weak solution of the Cauchy problem for the symmetric byperbolic system

$$
\begin{aligned}
& \frac{\partial}{\partial t} u+\sum_{i=1}^{n} \frac{\partial}{\partial x_{i}}\left(A^{i} u\right)+B u=0 \\
& u(x, 0)=\psi(x)
\end{aligned}
$$

under the assumption stated above. Furtber suppose that the following conditions (i), (ii) and (iii) are valid:

(i) The coefficients $A^{i}, B$ and their first derivatives in the distribution sense are bounded in $\mathfrak{D}$.

(ii) Let $\Delta t=t^{\prime}-t, \Delta x_{i}=x_{i}^{\prime}-x_{i}$, and $\Delta_{i} A^{j}=A^{j}\left(x_{1}, x_{2}, \cdots, x_{i}^{\prime}, \cdots, x_{n}, t\right)$ $-A^{j}\left(x_{1}, x_{2}, \cdots, x_{n}, t\right)$. Then there exist nonnegative locally integrable functions $\nu(t), \mu_{i}(t)(i=1,2, \cdots, n)$ bounded in $[0, \infty]$ such that, in $\mathfrak{D}_{\text {, }}$

$$
|\langle B \xi, \xi\rangle| \leq \nu(t)\langle\xi, \xi\rangle, \quad\left|\left\langle\frac{\Delta_{i} A^{i}}{\Delta_{i}} \xi, \xi\right\rangle\right| \leq \mu_{i}(t)\langle\xi, \xi\rangle \quad(i=1,2, \cdots, n)
$$

for any vector $\xi$.

(iii) For any compact set $\widetilde{H}_{t}$ in $H_{t}$, there exists a constant $K(>0)$ such that

$$
\int_{\tilde{H}_{t}}\langle u, u\rangle d x \leq K e^{-2 a t}, \quad t \in[0, \infty)
$$

for any constant $a>0$.

Then $u$ is identically equal to zero in $\mathfrak{D}$.

3. The energy inequality. We modify the coefficients of (4) by the standard mollifier method. Let $w_{k}^{(1)}(k=1,2, \ldots)$ be an infinitely differentiable nonnegative function on $E^{n}$ with support contained in $|x| \leq 1 / k$ and satisfying $\int_{E^{n}} w_{k}^{(1)}(x) d x=1$. Further, let $w_{k}^{(2)}(k=1,2, \ldots)$ be an infinitely differentiable function on $(-\infty, \infty)$ with compact support $|t| \leq 1 / k$ and satisfying $\int_{-\infty}^{\infty} w_{k}^{(2)}(t) d t=1$.

Denote by $w_{k}(y, s)$ the product $w_{k}^{(1)}(y) w_{k}^{(2)}(s)$. For any function $f$ locally integrable in $\mathfrak{D}$, we put

$$
f_{k}(x, t)=f * w_{k}(x, t)=\iint_{\mathscr{D}} f(y, s) w_{k}(x-y, t-s) d y d s
$$


Then $f_{k}(k=1,2, \ldots)$ is infinitely (many times) differentiable in $\mathscr{D}$ and $f_{k}$ tends to $f$ in the $L^{p}$-norm $(p \geq 1)$ on every compact subset of $\mathfrak{D}$ as $k$ tends to infinity.

We put $A_{k}(x, t)=\left(\overline{a_{i j}^{k}}(x, t)\right)$ for a matrix $A(x, t)=\left(a_{i j}(x, t)\right)$ with $a_{i j} \in L_{1 \text { oc }}^{2}(\mathscr{D})$, where $a_{i j}^{k}(x, t)=a_{i j} * w_{k}(x, t)$. In such a manner, we consider matrices $A_{k}^{i}$ $(i=1,2, \cdots, n)$ and $B_{k}$ for matrices $A^{i}(i=1,2,3, \cdots, n)$ and $B$ in $(4)$, respectively. Thus we have a system

$$
\frac{\partial}{\partial t} u+\sum_{i=1}^{n} \frac{\partial}{\partial x_{i}}\left(A_{k}^{i} u\right)+B_{k} u=0 .
$$

First we have the following

Lemma 1. The conditions in the Theorem imply that

(ii)' there exists a nonnegative function $\mu(t) \in L_{1 \text { oc }}^{1}([0, \infty))$ being bounded and satisfying

$$
\left|\left\langle\left(B_{k}+\frac{1}{2} \sum_{i=1}^{n} \frac{\partial A_{k}^{i}}{\partial x_{i}}\right) \xi, \xi\right\rangle\right| \leq \mu(t)\langle\xi, \xi\rangle
$$

for any $\xi$.

Proof. It is almost obvious that there exists $\nu_{0} \in L_{1 \text { oc }}^{1}([0, \infty))$ satisfying $\left|\left\langle B_{k} \xi, \xi\right\rangle\right| \leq \nu_{0}(t)\langle\xi, \xi\rangle$. In fact, we have

$$
\begin{aligned}
\left|\left\langle B_{k} \xi, \xi\right\rangle\right| & =\left|\sum_{i, j=1}^{l} b_{i j}^{k}(x, t) \xi_{i} \xi_{j}\right| \\
& =\left|\iint \sum_{i, j=1}^{l} b_{i j}(y, s) \xi_{i} \xi_{j} w_{k}(x-y, t-s) d y d s\right| \\
& \leq\left\langle\int_{E^{n}} w_{k}^{(1)}(x-y) d y \int_{0}^{\infty} \nu(s) w_{k}^{(2)}(t-s) d s \xi, \xi\right\rangle \\
& =\left\langle\int_{0}^{\infty} \nu(s) w_{k}^{(2)}(t-s) d s \xi, \xi\right\rangle=\nu_{0}(t)\langle\xi, \xi\rangle
\end{aligned}
$$

where $\nu_{0}(t)=\int_{0}^{\infty} \nu(s) w_{k}^{(2)}(t-s) d s \in L_{1 \text { oc }}^{1}([0, \infty))$ and bounded.

Next we have only to show that there exist nonnegative functions $\mu_{i}^{\prime}(t) \in L_{1 \text { oc }}^{1}([0, \infty))$ $(i=1,2, \cdots, n)$ being bounded such that

$$
\left|\wedge\left(\partial A_{k}^{i} / \partial x_{i}\right) \xi, \xi\right\rangle \mid \leq \mu_{i}^{\prime}(t)\langle\xi, \xi\rangle \quad(i=1,2,3, \cdots, n) .
$$

Since $\left\langle\left[\Delta_{i} A^{i} / \Delta x_{i}+\mu_{i}(t)\right] \xi, \xi\right\rangle \geq 0$ and since $w_{k}$ is positive, the convolution of $\left[\Delta_{i} A^{i} / \Delta x_{i}+\mu_{i}(t)\right]$ and $w_{k}$ is nonnegative, so $\left\langle\left[\Delta_{i} A^{i} / \Delta x_{i}+\mu_{i}\right] * w_{k}(x, t) \xi, \xi\right\rangle$ $\geq 0$ implies that 


$$
\left\langle\left[\Delta_{i} A_{k}^{i}(x, t) / \Delta x_{i}+\mu_{i}^{\prime}(t)\right] \xi, \xi\right\rangle \geq 0
$$

where $\mu_{i}^{\prime}(t)=\int_{0}^{\infty} \mu_{i}^{\prime}(t-s) w_{k}^{(2)}(s) d s \in L_{1 \text { oc }}^{1}([0, \infty))$ is bounded. By passing through the limit, with respect to the difference quotients we have

$$
\left\langle\left(\partial A_{k}^{i} / \partial x_{i}\right) \xi, \xi\right\rangle \geq-\mu_{i}^{\prime}(t)\langle\xi, \xi\rangle, \quad i=1,2,3, \cdots, n .
$$

Similarly, we have $\left(\left(\partial A_{k}^{i} / \partial x_{i}\right) \xi, \xi\right\rangle \leq \mu_{i}^{\prime}(t)\langle\xi, \xi\rangle$, and hence $\left|\left\langle\left(\partial A_{k}^{i} / \partial x_{i}\right) \xi, \xi\right\rangle\right|$ $\leq \mu_{i}^{\prime}(t)\langle\xi, \xi\rangle$. Thus putting $\mu(t)=\nu_{0}(t)+1 / 2 \Sigma_{i=1}^{n} \mu_{i}^{\prime}(t)$, we then obtain (ii)'. Q.E.D.

Lemma 2. If a function $A$ on $E^{n}$ and its derivatives in the distribution sense are bounded, then the smoothed functions $A_{k}$ and $\partial A_{k} / \partial x_{j}(j=1,2, \cdots, n)$ are uniformly bounded (independent of $k$ ), where $A_{k}=A * w_{k}$ and $w_{k}$ is a mollifier.

It is easy to prove this lemma, so we omit the proof here (cf. [6]).

Now consider the Cauchy problem

$$
\frac{\partial \phi}{\partial t}+\sum_{i=1}^{n} A_{k}^{i} \frac{\partial \phi}{\partial x_{i}}-B_{k}^{*} \phi=F,
$$

for any given function $F \in C^{\infty}(\mathfrak{D})$ with compact support. It is well known in the classical theory that the solution $\phi$ of (5) and (6) (depending on $k$ ) is sufficiently smooth and has compact support. This support is also independent of $k$. Here $A_{k}^{i}$ and $B_{k}^{*}$ are the smooth matrices associated with the matrices $A^{i}$ and the transpose $B^{*}$ of $B$ respectively.

By assuming (ii)' in Lemma 1 , we prove that for the solution $\phi_{k}$ of the Cauchy problem (5), (6), integrals $\int_{0}^{t} \int_{H_{s}}\left\langle\phi_{k}, \phi_{k}\right\rangle d x d s$ and $\int_{0}^{t} \int_{H_{s}}\left\langle\partial \phi_{k} / \partial x_{i}, \partial \phi_{k} / \partial x_{i}\right\rangle d x d s$ are bounded uniformly (independent of $k$ ). We start from the following lemma about the energy inequality.

Lemma 3. Let $\phi$ with compact support in $E^{n}$ be a solution of the Cauchy problem

$$
\begin{aligned}
& \frac{\partial \phi}{\partial t}+\sum_{i=1}^{n} A_{k}^{i} \frac{\partial \phi}{\partial x_{i}}-B_{k}^{*} \phi=F, \\
& \phi(x, 0)=\psi(x)
\end{aligned}
$$

for any given $\psi \in C_{0}^{\infty}\left(E^{n}\right)$ and $F \in C^{\infty}(\mathfrak{D})$ with compact support. Suppose that the coefficients of (5) satisfy the condition (ii)' in Lemma 1. Then the inequality

$$
\int_{H_{t}}\langle\phi, \phi\rangle d x+\int_{0}^{t} \int_{H_{s}}\langle\phi, \phi\rangle d x d s
$$

$$
\leq e^{2 \lambda t}\left(\int_{H_{0}}\langle\psi, \psi\rangle d x+\int_{0}^{t} \int_{H_{s}}\langle F, F\rangle d x d s\right)
$$


bolds for a sufficiently large $\lambda$ and for any $t \in[0, \infty)$.

Proof. It is obvious that

$$
\int_{0}^{t} \int_{H_{s}}\left\{\left\langle\frac{\partial \phi}{\partial t}, \phi\right\rangle+\sum_{i=1}^{n}\left\langle A_{k}^{i} \frac{\partial \phi}{\partial x_{i}}, \phi\right\rangle-\left\langle B_{k}^{*} \phi, \phi\right\rangle\right\} d x d s=\int_{0}^{t} \int_{H_{s}}\langle F, \phi\rangle d x d s
$$

implies

$$
\begin{aligned}
\int_{0}^{t} \int_{H_{s}}\left\{\left\langle\frac{\partial \phi}{\partial t}, \phi\right\rangle-\sum_{i=1}^{n}\left\langle\frac{\partial}{\partial x_{i}}\left(A_{k}^{i} \phi\right), \phi\right\rangle-\left\langle B_{k} \phi, \phi\right\rangle\right\} d x d s & \\
& =\int_{0}^{t} \int_{H_{s}}\langle F, \phi\rangle d x d s .
\end{aligned}
$$

By differentiating with respect to $t$, we have

(9) $\int_{H_{t}}\left\{-\left\langle\frac{\partial \phi}{\partial t}, \phi\right\rangle+\sum_{i=1}^{n}\left\langle\frac{\partial}{\partial x_{i}}\left(A_{k}^{i} \phi\right), \phi\right\rangle+\left\langle B_{k} \phi, \phi\right\rangle\right\} d x=\int_{H_{t}}\langle-F, \phi\rangle d x$.

It is clear that

$$
\int_{H_{t}}\left\langle\frac{\partial}{\partial x_{i}}\left(A_{k}^{i} \phi\right), \phi\right\rangle d x=\int_{H_{t}}\left\langle\left(\frac{\partial}{\partial x_{i}} A_{k}^{i}\right) \phi, \phi\right\rangle d x+\int_{H_{t}}\left\langle A_{k}^{i} \frac{\partial \phi}{\partial x_{i}}, \phi\right\rangle d x
$$

and

$$
\int_{H_{t}}\left\langle\frac{\partial}{\partial x_{i}}\left(A_{k}^{i} \phi\right), \phi\right\rangle d x=-\int_{H_{t}}\left\langle A_{k}^{i} \phi, \frac{\partial \phi}{\partial x_{i}}\right\rangle d x=-\int_{H_{t}}\left\langle A_{k}^{i} \frac{\partial \phi}{\partial x_{i}}, \phi\right\rangle d x
$$

so we have

$$
-\frac{1}{2} \int_{H_{t}} \sum_{i=1}^{n}\left\langle\frac{\partial A_{k}^{i}}{\partial x_{i}} \phi, \phi\right\rangle d x=\int_{H_{t}} \sum_{i=1}^{n}\left\langle A_{k}^{i} \frac{\partial \phi}{\partial x_{i}}, \phi\right\rangle d x
$$

Since $\phi \in C^{\infty}(\mathscr{D})$ has compact support in $E^{n}$, we see that

$$
\frac{d}{d t} \int_{H_{t}}\langle\phi, \phi\rangle d x=2 \int_{H_{t}}\left\langle\frac{\partial \phi}{\partial t}, \phi\right\rangle d x .
$$

Set $\phi=e^{\lambda t} v$ and $f=+e^{-\lambda t} F$ for a constant $\lambda(>0)$. Then (10) and (11) are satisfied by $v$ instead of $\phi$ and we have 


$$
\begin{aligned}
& \int_{H_{t}}\left\langle\frac{\partial v}{\partial t}, v\right\rangle d x \\
& \quad=\int_{H_{t}}\left\{+\left\langle\sum_{i=1}^{n} A_{k}^{i} \frac{\partial v}{\partial x_{i}}, v\right\rangle+\left\langle\sum_{i=1}^{n} \frac{\partial A_{k}^{i}}{\partial x_{i}} v, v\right\rangle+\left\langle\left(B_{k}-\lambda I\right) v, v\right\rangle+\langle f, v\rangle\right\} d x,
\end{aligned}
$$

which is equivalent to (9), where $I$ denotes the identity matrix. Applying the identity (10), we then have

$$
2 \int_{H_{t}}\left\langle\frac{\partial v}{\partial t}, v\right\rangle d x=2 \int_{H_{t}}\langle f, v\rangle d x+\int_{H_{t}}\left\langle\left[2\left(B_{k}-\lambda I\right)+\sum_{i=1}^{n} \frac{\partial A_{k}^{i}}{\partial x_{i}}\right] v, v\right\rangle d x .
$$

Therefore, by the virtue of (11), we get

$$
\frac{d}{d t} \int_{H_{t}}\langle v, v\rangle d x=2 \int_{H_{t}}\langle f, v\rangle d x+\int_{H_{t}}\left\langle\left[2\left(B_{k}-\lambda I\right)+\sum_{i=1}^{n} \frac{\partial A_{k}^{i}}{\partial x_{i}}\right] v, v\right\rangle d x_{0}
$$

Integrating this identity with respect to $t$ from 0 to $t$, we have

$$
\begin{aligned}
\int_{H_{t}}\langle v, v\rangle d x-\int_{H_{0}}\langle v, v\rangle d x \\
=2 \int_{0}^{t} \int_{H_{s}}\langle f, v\rangle d x d s+\int_{0}^{t} \int_{H_{s}}\left\langle\left(2 B_{k}+\sum_{i=1}^{n} \frac{\partial A_{k}^{i}}{\partial x_{i}}\right) v, v\right\rangle d x \\
\quad-2 \lambda \int_{0}^{t} \int_{H_{s}}\langle v, v\rangle d x d s .
\end{aligned}
$$

By $v(x, 0)=\phi(x, 0)=\psi(x)$, we have

$$
\begin{aligned}
\int_{H_{t}}\langle & v, v\rangle d x-\int_{H_{0}}\langle\psi, \psi\rangle d x \\
\leq & \int_{0}^{t} \int_{H_{s}}|f|^{2} g^{-2}(s) d x d s+\int_{0}^{t} \int_{H_{s}}|v|^{2} g^{2}(s) d x d s \\
& +\int_{0}^{t} \int_{H_{s}}\left\langle\left[2 B_{k}+\sum_{i=1}^{n} \frac{\partial A_{k}^{i}}{\partial x_{i}}\right] v, v\right\rangle d x d s-2 \lambda \int_{0}^{t} \int_{H_{s}}\langle v, v\rangle d x d s
\end{aligned}
$$

where we assume that $g(t)(\neq 0)$ together with $g^{-1}(t)$ is an arbitrary locally' square integrable function on $[0, \infty)$. Therefore we have 


$$
\begin{aligned}
& \int_{H_{t}}\langle v, v\rangle d x+\int_{0}^{t} \int_{H_{s}}\langle v, v\rangle d x d s \\
& \leq \int_{H_{0}}\langle\psi, \psi\rangle d x+\int_{0}^{t} \int_{H_{s}} g^{-2}(s)|f|^{2} d x d s \\
& \quad+\int_{0}^{t} \int_{H_{s}}\left[g^{2}(s)+1-2 \lambda\right]|\cdot v|^{2} d x d s+\int_{0}^{t} \int_{H_{s}}\left\langle\left(2 B_{k}+\sum_{i=1}^{n} \frac{\partial A_{k}^{i}}{\partial x_{i}}\right) v, v\right\rangle d x d s .
\end{aligned}
$$

Choose $g(t)$ such that $g^{2}(t)=2 \lambda-2 \mu(t)-1 \geq 1$ for a sufficiently large $\lambda$, where $\mu(t)$ is such a function as in Lemma 1 . Then

$\int_{H_{t}}\langle v, v\rangle d x+\int_{0}^{t} \int_{H_{s}}\langle v, v\rangle d x d s \leq \int_{H_{0}}\langle\psi, \psi\rangle d x+\int_{0}^{t} \int_{H_{s}} g^{-2}(s)|f|^{2} d x d s$ or

$$
\begin{aligned}
\int_{H_{t}} & \langle\phi, \phi\rangle d x+\int_{0}^{t} \int_{H_{s}}\langle\phi, \phi\rangle d x d s \\
& \leq e^{2 \lambda t}\left[\int_{H_{0}}\langle\psi, \psi\rangle d x+\int_{0}^{t} \int_{H_{s}}\langle F, F\rangle d x d s\right] .
\end{aligned}
$$

This proves our lemma.

An analogous inequality is satisfied by energy integrals $\|u(t)\|_{1}$ defined as

$$
\|u(t)\|_{1}=\left(\int_{H_{t}}\left(|u|^{2}+\left|D^{1} u\right|^{2}\right) d x\right)^{1 / 2},
$$

where $D^{1} u=\sum_{i=1}^{n} \partial u / \partial x_{i}$ and $\left|D^{1} u\right|^{2}=\sum_{i=1}^{n}\left|\partial u / \partial x_{i}\right|^{2}$. It follows that

Lemma 4. Under the assumption in the Theorem, the solution $\phi$ of (5), (7) with compact support satisfies the following inequality:

$$
\int_{H_{t}}\left(|\phi|^{2}+\left|D^{1} \phi\right|^{2}\right) d x+\int_{0}^{t} \int_{H_{s}}\left(|\phi|^{2}+\left|D^{1} \phi\right|^{2}\right) d x d s
$$

$$
\leq C e^{4 \lambda t}\left[\int_{H_{0}}\left(|\psi|^{2}+\left|D^{1} \psi\right|^{2}\right) d x+\int_{0}^{t} \int_{H_{s}}\left(|F|^{2}+\left|D^{1} F\right|^{2}\right) d x d s\right]
$$

or simply denoted by

$$
\|\phi(t)\|_{1}^{2}+\int_{0}^{t}\|\phi(s)\|_{1}^{2} d s \leq C e^{4 \lambda t}\left(\|\psi\|_{1}^{2}+\int_{0}^{t}\|F(s)\|_{1}^{2} d s\right)
$$

where $C$ is a constant and $\lambda$ is sufficiently large.

Proof. Recall the equation

$$
L \phi \equiv \sum_{i=1}^{n} A_{k}^{i} \frac{\partial \phi}{\partial x_{i}}+\frac{\partial \phi}{\partial t}-B_{k}^{*} \phi=F .
$$


Differentiating (5) with respect to $x_{j}$, we have

$$
L \frac{\partial \phi}{\partial x_{j}}+\sum_{i=1}^{n} \frac{\partial \phi}{\partial x_{i}} \cdot \frac{\partial A_{k}^{i}}{\partial x_{j}}-\frac{\partial B_{k}^{*}}{\partial x_{j}} \phi=\frac{\partial F}{\partial x_{j}} .
$$

For brevity, we put $V=\Sigma_{i=1}^{n} \partial \phi / \partial x_{j}=D^{1} \phi$ and then

$$
L V=D^{1} F+D^{1} B_{k}^{*} \cdot \phi-\sum_{i=1}^{n} \frac{\partial \phi}{\partial x_{i}} \cdot D^{1} A_{k}^{i} \equiv b, \text { say. }
$$

It is evident that $b$ is continuous and has compact support, thus by the same argument as in the proof of Lemma 3 , we obtain

$$
\begin{aligned}
& \int_{H_{t}}\langle U, U\rangle d x-\int_{H_{0}}\langle U, U\rangle d x \\
& =2 \int_{0}^{t} \int_{H_{s}}\left\langle e^{-\lambda s} b, U\right\rangle d x d s \\
& \quad+\int_{0}^{t} \int_{H_{s}}\left\langle\left(2 B_{k}+\sum_{i=1}^{n} \frac{\partial A_{k}^{i}}{\partial x_{i}}\right) U, U\right\rangle d x d s-2 \lambda \int_{0}^{t} \int_{H_{s}}\langle U, U\rangle d x d s,
\end{aligned}
$$

where $U=e^{-\lambda t} V(\lambda>0)$. Clearly we see

$$
\begin{aligned}
\int_{H_{t}}\langle U, U\rangle d x-\int_{H_{0}}\langle U, U\rangle d x \\
\leq 2 \int_{0}^{t} \int_{H_{s}}\left\langle e^{-\lambda s} h, U\right\rangle d x d s+\int_{0}^{t} \int_{H_{s}}\langle(2 \mu(s)-2 \lambda) U, U\rangle d x d s \\
=2 \int_{0}^{t} \int_{H_{s}} e^{-\lambda s}\left\langle\left(D^{1} F+D^{1} B_{k}^{*} \phi-\sum_{i=1}^{n} \frac{\partial \phi}{\partial x_{i}} D^{1} A_{k}^{i}\right), U\right\rangle d x d s \\
+\int_{0}^{t} \int_{H_{s}}\langle(2 \mu(s)-2 \lambda) U, U\rangle d x d s \\
\leq \int_{0}^{t} \int_{H_{s}}\left|D^{1} F\right|^{2} g^{-2}(s) d x d s+\int_{0}^{t} \int_{H_{s}}\left|D^{1} B_{k}^{*} \phi\right|^{2} g^{-2}(s) d x d s \\
+\int_{0}^{t} \int_{H_{s}} e^{-2 \lambda s} \sum_{i=1}^{n}\left|\frac{\partial \phi}{\partial x_{i}}\right|^{2}\left|D^{1} A_{k}^{i}\right|^{2} \cdot g^{-2}(s) d x d s \\
+\int_{0}^{t} \int_{H_{s}}\left(3 g^{2}(s)-2 \lambda+2 \mu(s)\right)|U|^{2} d x d s,
\end{aligned}
$$

where $g(t)$ is locally integrable and nowhere zero in $[0, \infty)$. Let $M$ be the bound 
of $D^{1} A_{k}^{i}(i=1,2, \cdots, n)$ and $C$ the bound of $D^{1} B_{k}^{*}$. It is known that $M$ and $C$ are independent of $k$. Choose $g^{2}(s)=(1 / 3)[2 \lambda-2 \mu(s)-1-M] \geq 1$ for a sufficiently large $\lambda$. Then

$$
\begin{aligned}
\int_{H_{t}}\langle U, U\rangle d x+(1+M) \int_{0}^{t} \int_{H_{s}}\langle U, U\rangle d x d s \\
\leq \int_{H_{0}}\langle U, U\rangle d x+\int_{0}^{t} \int_{H_{s}}\left\langle D^{1} F, D^{1} F\right\rangle d x d s \\
\quad+C \int_{0}^{t} \int_{H_{s}}\langle\phi, \phi\rangle d x d s+M \int_{0}^{t} \int_{H_{s}} e^{-2 \lambda s} \sum_{i=1}^{n}\left\langle\frac{\partial \phi}{\partial x_{i}}, \frac{\partial \phi}{\partial x_{i}}\right\rangle d x d s
\end{aligned}
$$

or

$$
\begin{aligned}
& \int_{H_{t}}\left|D^{1} \phi\right|^{2} d x+e^{2 \lambda t} \int_{0}^{t} \int_{H_{s}} e^{-2 \lambda s}\left|D^{1} \phi\right|^{2} d x d s \\
& \quad \leq e^{2 \lambda t}\left\{\int_{H_{0}}\left|D^{1} \psi\right|^{2} d x+\int_{0}^{t} \int_{H_{s}}\left|D^{1} F\right|^{2} d x d s+C \int_{0}^{t} \int_{H_{s}}|\phi|^{2} d x d s\right\} .
\end{aligned}
$$

Thus

$$
\int_{H_{t}}\left|D^{1} \phi\right|^{2} d x+\int_{0}^{t} \int_{H_{s}}\left|D^{1} \phi\right|^{2} d x d s
$$

$$
\leq e^{2 \lambda t}\left\{\int_{H_{0}}\left|D^{1} \psi\right|^{2} d x+\int_{0}^{t} \int_{H_{s}}\left|D^{1} F\right|^{2} d x d s+C \int_{0}^{t} \int_{H_{s}}|\phi|^{2} d x d s\right\} .
$$

Multiplying (8) by $\left(1+C e^{2 \lambda t}\right)$ and then adding to (15), we obtain

$$
\begin{aligned}
& \int_{H_{t}}\left(|\phi|^{2}+\left|D^{1} \phi\right|^{2}\right) d x+\int_{0}^{t} \int_{H_{s}}\left(|\phi|^{2}+\left|D^{1} \phi\right|^{2}\right) d x d s+C e^{2 \lambda t} \int_{H_{s}}|\phi|^{2} d x \\
& \leq e^{2 \lambda t}\left\{\int_{H_{0}}\left(|\psi|^{2}+\left|D^{1} \psi\right|^{2}\right) d x+\int_{0}^{t} \int_{H_{s}}\left(|F|^{2}+\left|D^{1} F\right|^{2}\right) d x d s\right\} \\
& \quad+C e^{4 \lambda t}\left\{\int_{H_{0}}|\psi|^{2} d x+\int_{0}^{t} \int_{H_{s}}|F|^{2} d x d s\right\} .
\end{aligned}
$$

Therefore,

$$
\begin{aligned}
& \int_{H_{t}}\left(|\phi|^{2}+\left|D^{1} \phi\right|^{2}\right) d x+\int_{0}^{t} J_{H_{s}}\left(|\phi|^{2}+\left|D^{1} \phi\right|^{2}\right) d x d s \\
& \quad \leq M_{t}\left\{\int_{H_{0}}\left(|\psi|^{2}+\left|D^{1} \psi\right|^{2}\right) d x+\int_{0}^{t} \int_{H_{s}}\left(|F|^{2}+\left|D^{1} F\right|^{2}\right) d x d s\right\}
\end{aligned}
$$

where $M_{t}=\max \left(e^{2 \lambda t}, C e^{4 \lambda t}\right)$. The constant $C$ can be taken as larger than 1 , so $M_{t}$ is chosen as $M_{t}=C e^{4 \lambda t}$.

4. Proof of the main theorem. The weak solution $u$ of the equation (4) satisfies the following equality: 
1972]

WEAK SOLUTIONS FOR HYPERBOLIC SYSTEMS

435

$$
\begin{aligned}
\int_{0}^{t} \int_{H_{s}}\left[\sum_{i=1}^{n}\left\langle A^{i} u, \frac{\partial \phi}{\partial x_{i}}\right\rangle+\left\langle u, \frac{\partial \phi}{\partial s}\right\rangle-\langle B u, \phi\rangle\right] d x d s & \\
& +\int_{H_{0}}\langle u, \phi\rangle d x-\int_{H_{t}}\langle u, \phi\rangle d x=0
\end{aligned}
$$

for any $\phi \in C^{\infty}(\mathscr{D})$ with compact support in $\mathscr{D}$ and for any fixed $t \in[0, \infty)$. We have to prove that $u$ vanishes indentically in $\mathscr{D}$ under our assumption. It sufficies to show that $\int_{0}^{\infty} \int_{H_{s}}\langle u, F\rangle d x d t=0$ for any $F \in C^{\infty}(\mathcal{D})$ with compact support in D. Let $\phi_{k}$ be the solution of (5), (6). Then (16) holds for $\phi=\phi_{k}$.

By (5) and (16),

$$
\begin{aligned}
\int_{0}^{t} & \int_{H_{s}}\langle u, F\rangle d x d s=\int_{0}^{t} \int_{\tilde{H}_{s}}\langle u, F\rangle d x d s \\
= & \int_{0}^{t} \int_{\tilde{H}_{s}}\left\langle u, \sum_{i=1}^{n} A_{k}^{i} \frac{\partial \phi_{k}}{\partial x_{i}}+\frac{\partial \phi_{k}}{\partial s}-B_{k}^{*} \phi_{k}\right\rangle d x d s \\
& -\int_{0}^{t} \int_{\tilde{H}_{s}}\left\langle u, \sum_{i=1}^{n} A^{i} \frac{\partial \phi_{k}}{\partial x_{i}}+\frac{\partial \phi_{k}}{\partial s}-B^{*} \phi_{k}\right\rangle d x d s \\
& -\int_{\tilde{H}_{0}}\left\langle u, \phi_{k}\right\rangle d x+\int_{\tilde{H}_{t}}\left\langle u, \phi_{k}\right\rangle d x,
\end{aligned}
$$

where $\widetilde{H}_{t}$ is the support of $F$ in $H_{t}$. Since $\phi_{k}$ vanishes on the hyperplane $H_{0}$ and has compact support in $H_{t}$, we have

$$
\begin{aligned}
\left|\int_{0}^{t} \int_{H_{s}}\langle u, F\rangle d x d s\right| \\
\quad \leq \int_{0}^{t} \int_{H_{s}}\left|\left\langle u, \sum_{i=1}^{n}\left(A_{k}^{i}-A^{i}\right) \frac{\partial \phi_{k}}{\partial x_{i}}\right\rangle\right| d x d s \\
\quad+\int_{0}^{t} \int_{H_{s}}\left|\left\langle u,\left(B_{k}^{*}-B^{*}\right) \phi_{k}\right\rangle\right| d x d s+\int_{H_{s}}\left|\left\langle u, \phi_{k}\right\rangle\right| d x \\
=\int_{0}^{t} \int_{\tilde{H}_{s}}\left|\left\langle u, \sum_{i=1}^{n}\left(A_{k}^{i}-A^{i}\right) \frac{\partial \phi_{k}}{\partial x_{i}}\right\rangle\right| d x d s \\
\quad+\int_{0}^{t} \int_{\tilde{H}_{s}}\left|\left\langle u,\left(B_{k}^{*}-B^{*}\right) \phi_{k}\right\rangle\right| d x d s+\int_{\tilde{H}_{t}}\left|\left\langle u, \phi_{k}\right\rangle\right| d x .
\end{aligned}
$$

It follows from Lemma 3 and Lemma 4 that

$$
\int_{0}^{t} \int_{\tilde{H}_{s}}\left\langle\phi_{k}, \phi_{k}\right\rangle d x d s \text { and } \int_{0}^{t} \int_{\tilde{H}_{s}} \sum_{i=1}^{n}\left\langle\frac{\partial \phi_{k}}{\partial x_{i}}, \frac{\partial \phi_{k}}{\partial x_{i}}\right\rangle d x d s
$$


are uniformly bounded (independent of $k$ ). On the other hand, $A_{k}^{i} \rightarrow A^{i} \quad(i=1,2$, $\cdots, n)$ and $B_{k}^{*} \rightarrow B^{*}(k \rightarrow \infty)$ in $L^{2}$-norm on $\widetilde{H}_{t} \subset \mathcal{D}$. Therefore, for $u \in L_{\text {loc }}^{2}(\mathfrak{D})$,

$$
\begin{aligned}
\int_{0}^{t} \int_{\tilde{H}_{s}} \mid\langle u, & \left.\sum_{i=1}^{n}\left(A_{k}^{i}-A_{i}\right) \frac{\partial \phi_{k}}{\partial x_{i}}\right\rangle \mid d x d s \\
& \leq\|u\|_{L^{2}\left(\tilde{H}_{t}\right)}\left\{\sum_{i=1}^{n} \int_{0}^{t} \int_{\tilde{H}_{s}}\left|\left(A_{k}^{i}-A^{i}\right) \frac{\partial \phi_{k}}{\partial x_{i}}\right|^{2} d x d s\right\}^{1 / 2} \\
& \rightarrow 0 \text { as } k \rightarrow \infty
\end{aligned}
$$

and

$$
\begin{aligned}
\int_{0}^{t} \int_{\tilde{H}_{s}}\left|\left\langle u,\left(B_{k}^{*}-B^{*}\right) \phi_{k}\right\rangle\right| d x d s & \leq\|u\|_{L 2\left(\tilde{H}_{t}\right)}\left(\int_{0}^{t} \int_{\tilde{H}_{s}}\left|\left(B_{k}^{*}-B^{*}\right) \phi_{k}\right|^{2} d x d s\right)^{1 / 2} \\
& \rightarrow 0 \text { as } k \rightarrow \infty .
\end{aligned}
$$

By (iii) the last term on the right-hand side of (17) can be estimated as follows:

$$
\int_{\tilde{H}_{t}}\left|\left\langle u, \phi_{k}\right\rangle\right| d x \leq\left\|\phi_{k}\right\|_{L^{2}\left(\tilde{H}_{t}\right)}\left(\int_{\tilde{H}_{t}}|u|^{2} d x\right)^{1 / 2} \leq\left\|\phi_{k}\right\|_{L^{2}\left(\tilde{H}_{t}\right)} \cdot\left(K e^{-2 a t}\right)^{1 / 2}
$$

for any positive $a$. Therefore,

$$
\left|\int_{0}^{t} \int_{H_{s}}\langle u, F\rangle d x d s\right| \leq K^{1 / 2}\left\|\phi_{k}\right\|_{L^{2}\left(\tilde{H}_{t}\right)} e^{-a t},
$$

which together with Lemma 3 yields

$$
\lim _{t \rightarrow \infty}\left|\int_{0}^{t} \int_{H_{s}}\langle u, F\rangle d x d s\right|=0 .
$$

This proves that the weak solution $u$ vanishes identically on $\mathscr{D}$.

\section{REFERENCES}

1. E. D. Conway, Generalized solutions of linear differential equations with discontinuous coefficients and the uniqueness question for multidimensional quasilinear conservation laws, J. Math. Anal. Appl. 18 (1967), 238-251. MR 34 \#6293.

2. R. Courant and D. Hilbert, Methods of mathematical physics. Vol. 2: Partial differential equations (Vol. 2 by R. Courant), Interscience, New York, 1962. MR 25 \#4216.

3. I. M. Gel'fand, Some questions of analysis and differential equations, Uspehi Mat. Nauk 14 (1959), no. 3 (87), 3-19; English transl., Amer. Math. Soc. Transl. (2) 26 (1963), 201-219. MR 22 \#12294; MR $27 \# 1694$.

4. A. E. Hurd and D. H. Sattinger, Questions of existence and uniqueness for hyperbolic equations with discontinuous coefficients, Trans. Amer. Math. Soc. 132 (1968), 159-174. MR 36 \#5509.

5. Kyuya Masuda, On the exponential decay of solutions for some partial differential equations, J. Math. Soc. Japan 19 (1967), 82-90. MR 34 \#4663.

6. S. L. Sobolev, Applications of functional analysis in mathematical physics, Izdat. Leningrad. Gos. Univ., Leningrad, 1950; English transl., Transl. Math. Monographs, vol. 7, Amer. Math. Soc., Providence, R. I., 1963. MR 14, 565; MR 29 \#2624.

INSTITUTE OF MATHEMATICS, NATIONAL TSING HUA UNIVERSITY, TAIWAN, REPUBLIC OF CHINA 